\title{
Effect of scaffold architecture and BMP-2/BMP-7 delivery on in vitro bone regeneration
}

\author{
Pinar Yilgor $\cdot$ Rui A. Sousa $\cdot$ Rui L. Reis $\cdot$ \\ Nesrin Hasirci • Vasif Hasirci
}

Received: 12 March 2010/ Accepted: 7 August 2010/Published online: 26 August 2010

(C) Springer Science+Business Media, LLC 2010

\begin{abstract}
The aim of this study was to develop 3-D tissue engineered constructs that mimic the in vivo conditions through a self-contained growth factor delivery system. A set of nanoparticles providing the release of BMP-2 initially followed by the release of BMP-7 were incorporated in poly( $\varepsilon$-caprolactone) scaffolds with different 3-D architectures produced by 3-D plotting and wet spinning. The release patterns were: each growth factor alone, simultaneous, and sequential. The orientation of the fibers did not have a significant effect on the kinetics of release of the model protein BSA; but affected proliferation of bone marrow mesenchymal stem cells. Cell proliferation on random scaffolds was significantly higher compared to the oriented ones. Delivery of BMP-2 alone suppressed MSC proliferation and increased the ALP activity to a higher level than that with BMP-7 delivery. Proliferation rate was suppressed the most by the sequential delivery of the two
\end{abstract}

P. Yilgor · N. Hasirci · V. Hasirci

METU, BIOMAT, Department of Biotechnology, Biotechnology

Research Unit, 06531 Ankara, Turkey

R. A. Sousa - R. L. Reis

3B's Research Group-Biomaterials, Biodegradables and Biomimetics, IBB-Institute for Biotechnology and

Bioengineering, PT Associated Laboratory, Headquarters of the European Institute of Excellence on Tissue Engineering and Regenerative Medicine, University of Minho, AvePark, Taipas, 4806-909 Guimaraes, Portugal

N. Hasirci

METU, BIOMAT, Faculty of Arts and Sciences, Department of Chemistry, 06531 Ankara, Turkey

V. Hasirci $(\bowtie)$

METU, BIOMAT, Department of Biological Sciences, Biotechnology Research Unit, 06531 Ankara, Turkey

e-mail: vhasirci@metu.edu.tr growth factors from the random scaffold on which the ALP activity was the highest. Results indicated the distinct effect of scaffold architecture and the mode of growth factor delivery on the proliferation and osteogenic differentiation of MSCs, enabling us to design multifunctional scaffolds capable of controlling bone healing.

\section{Introduction}

Bone can repair itself but it has been recognized that this has not always been satisfactory. Especially in the case of large defects a filler is required to temporarily fill the site over which the new tissue forms. The ideal bone filler or substitute is expected to satisfy some essential requirements such as being osteoconductive, mimicking the porosity and microstructure of the natural tissue, having the ability to harbor osteoinductive factors and osteogenic cells. Among all the bone substitutes currently in use in the clinic including biological (autogenic, allogenic, or xenogenic grafts, demineralized bone matrix, cadaver bone) and synthetic (metallic, ceramic, or polymeric) grafts, the only one which has all the required properties is the autograft. However, due to donor site morbidity, risk of infection, etc. the search for alternative therapies is continuing. A serious alternative to the autograft is growth factor releasing tissue engineered constructs. Tissue engineered bone substitutes consist of bone forming cells, porous scaffolds that serve as the microenvironment, and factors that guide proliferation and differentiation of cells. A multifunctional construct that combines a local bioactive agent delivery system with an osteoconductive scaffold and stem cells would be ideal. Such a graft would serve as an artificial ECM that combines osteoconduction and osteoinduction and protects the activity and maintains the local dose of the biological cues. 
Scaffold design is one of the crucial steps of tissue engineering approach. Rapid prototyping (RP) can produce a scaffold directly from computed tomography (CT) or magnetic resonance imaging (MRI) scans of the defect site or from a computer design of an irregular defect site [1-3]. Although RP has its own drawbacks such as the requirement of a specific form of input material (e.g. filament, powder, pellet, solution), the resolution (ca. $100 \mu \mathrm{m}$ ) that limits the detail of the produced construct, the morphology which generally presents edges and corners, the technique still holds great promise for the production of custom-made scaffolds and prostheses. 3-D plotting is the most convenient method for bone tissue engineering due to its milder operation conditions, absence of left over polymer powder within the scaffold and the ability to produce scaffolds without any binders.

During bone regeneration, a variety of growth factors including transforming growth factor-beta (TGF- $\beta$ ) superfamily proteins (especially bone morphogenetic proteins, BMPs), insulin-like growth factor (IGF), fibroblast growth factor (FGF), platelet-derived growth factor (PDGF) and vascular endothelial growth factor (VEGF) function with a complex time and concentration pattern [4]. Among these, BMPs were shown to induce bone formation by inducing mesenchymal stem cells (MSCs) toward osteoblastic differentiation and are the most osteogenic growth factors presently described [5-7]. Some studies combined BMPs with other growth factors to achieve improved repair. For example, BMP-2 and TGF- $\beta 3$ were combined and their delivery led to enhanced bone formation in vivo while the effect of a single growth factor was negligible [8]. The positive effects of VEGF combined with BMP-2 on in vivo bone regeneration $[9,10]$ and IGF-1 jointly encapsulated in microparticles with BMP-2 and then loaded in porous scaffolds for in vitro periodontal regeneration were also reported [11]. BMP-7 was shown to couple with BMP-2 in inducing bone morphogenesis and both were approved by FDA for use in clinical applications [12-15]. Therefore, it was considered that developing scaffolds that could deliver a combination of BMP-2 with BMP-7 in a time dependent manner is a viable biomimetic approach towards bone healing. Our previous studies illustrated that delivering BMP-2 and BMP-7 in a sequential manner in both free nanoparticulate form [16] and after incorporation into scaffolds [17, 18] enhance osteogenic differentiation of MSCs in vitro.

In this study, poly( $\varepsilon$-caprolactone) (PCL) scaffolds were produced by $3-\mathrm{D}$ plotting and wet spinning and tested for in vitro bone regeneration. Nanoparticles carrying BMP-2 and BMP-7 were added into these scaffolds to produce a multifunctional construct. The effect of scaffold geometry and growth factor delivery from the scaffolds were investigated using bone marrow MSCs.

The multifunctional scaffolds prepared in this study could be used in all types of congenital and acquired fractures and defects to achieve enhanced and accelerated healing through the self-contained sequential growth factor delivery system. These scaffolds are especially suitable for use in large bone defects, non-unions, comminuted and osteoporotic fractures that need osteoinduction as well as structural and mechanical support.

\section{Materials and methods}

\subsection{Materials}

PCL $\left(\right.$ Mw $\left.3.7 \times 10^{4}\right)$ was purchased from Solvay Caprolactones (CAPA 6404; UK). Poly(lactic acid-co-glycolic acid) (PLGA) (50:50) (Resomer ${ }^{\circledR}$ RG503H) (i.v. 0.32-0.44 dl/g, $0.1 \%$ in chloroform, $25^{\circ} \mathrm{C}$ ) was purchased from BoehringerIngelheim (Germany). Poly(3-hydroxybutyrate-co-3-hydroxyvalerate) (PHBV) (HV content $8 \% \mathrm{w} / \mathrm{w})$, dexamethasone, $\beta$-glycerophosphate disodium salt, L-ascorbic acid were bought from Sigma-Aldrich (Germany). Bovine serum albumin (BSA) and polyvinyl alcohol (PVA) (Mw $1.5 \times 10^{4}$ ) were obtained from Fluka (USA). Recombinant human BMP-2 from InductOs ${ }^{\circledR}$ (Wyeth Pharmaceuticals, USA) and recombinant human BMP-7 from Prospec Tany Technogene (Israel) were used. Dulbecco's Modified Eagle Medium (DMEM, high glucose) and fetal bovine serum (FBS) were obtained from Hyclone (USA). NucleoCounter reagents were supplied by Chemometec (Denmark) and Alamar Blue cell proliferation assay was from Biosource (USA). For the assessment of cell differentiation, alkaline phosphatase kit (Randox, USA) was used.

\subsection{Preparation of BSA, BMP-2 and BMP-7 loaded nanocapsules}

PLGA and PHBV nanocapsules containing BMP-2 and BMP-7, respectively, or BSA in both type of nanocapsules, were prepared by the w/o/w double emulsion technique as reported earlier [16]. Briefly, an aqueous solution of BSA or BMP was emulsified in a dichloromethane solution of PLGA or PHBV and this was then introduced to a larger volume of aqueous PVA solution. Nanocapsules were collected by centrifugation, washed with Tris- $\mathrm{HCl}$ ( $\mathrm{pH} 7.4$ ), resuspended in the buffer and dried with lyophilization.

\subsection{Production of PCL scaffolds}

\subsubsection{3-D plotting}

Oriented PCL scaffolds were fabricated using a Bioplotter ${ }^{\circledR}$ (Envisiontec GmbH, Germany) [19]. Before the plotting procedure, PCL cartridges were prepared by manually 


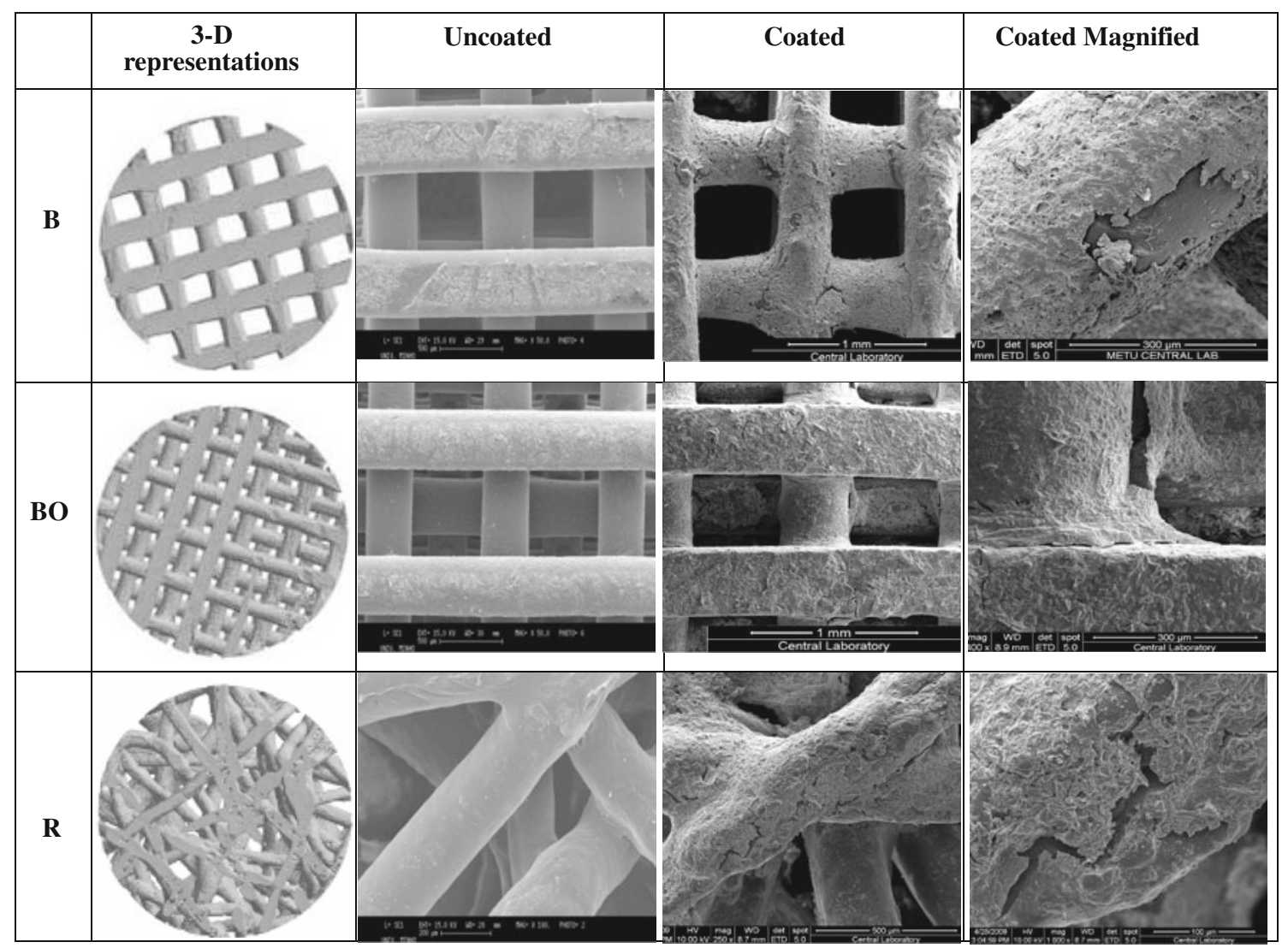

Fig. 13 -D representations of PCL scaffolds (left column) and SEM of PLGA nanocapsules incorporated onto the fiber surfaces of PCL scaffolds (uncoated, coated and coated magnified)

compressing ca. $5 \mathrm{~g}$ of polymer in plastic tubes while heating to $100^{\circ} \mathrm{C}$ until the polymer slightly melts. After cooling, the cartridges were removed from the holder, placed in the stainless steel syringe of the Bioplotter and brought to $140^{\circ} \mathrm{C}$ in the heated cartridge unit. Meanwhile, rectangular block models $(20 \mathrm{~mm} \times 20 \mathrm{~mm})$ were uploaded on the Bioplotter CAD/CAM software. When the polymer melted, $\mathrm{CO}_{2}$ pressure $(5 \mathrm{~mm} \mathrm{Hg})$ was applied to the syringe through a pressurized cap, and 3-D scaffolds were plotted up to 10 layers by extrusion of polymer fibers. Each layer was $20 \mathrm{~mm} \times 20 \mathrm{~mm}$ with a thickness of $0.25 \mathrm{~mm}$ yielding a final 10 layered scaffold of $20 \times 20 \times$ $2.5 \mathrm{~mm}$.

Scaffolds with different architectures were produced by changing the respective orientation of the deposited fibers using the Bioplotter's CAD/CAM software. PCL scaffolds with two different standard architectures were produced: basic (B), basic-offset (BO). The B architecture (Fig. 1) had each layer $(\mathrm{N})$ orthogonal to the layers below $(\mathrm{N}-1)$, and above $(\mathrm{N}+1)$. Here $\mathrm{N}-1$ and $\mathrm{N}+1$ were exactly in the same $\mathrm{x}-\mathrm{y}$ position. The $\mathrm{BO}$ architecture (Fig. 1) was similar to $\mathrm{B}$, but $\mathrm{N}-1$ and $\mathrm{N}+1$ layers were offset by a given distance in the $x-y$ plane. Finally, the scaffolds were cut using a circular die with $5 \mathrm{~mm}$ diameter.

\subsubsection{Wet spinning}

PCL scaffolds with randomly oriented fibers were prepared by wet spinning. A polymer paste was made in chloroform (90\%, w/v) which was then loaded into a syringe pump (World Precision Instruments, UK), and extruded through a needle (i.d. $0.5 \mathrm{~mm}$ ) into cold methanol at a rate of $6 \mathrm{ml} / \mathrm{h}$. Randomly oriented fibers formed upon manual movement of the coagulation bath in the $x-y$ plane. In order to prevent fusion of the fibers in the various layers, separate layers of ca. $0.8 \mathrm{~mm}$ thickness were produced from $0.2 \mathrm{ml}$ polymer paste, the layers were washed with distilled water and dried overnight at room temperature. Then, 3 of these layers were moistened with hexane:chloroform $(4: 1 \mathrm{v} / \mathrm{v})$ and manually pressed together to form 3-D random structure (R) (Fig. 1).

\subsection{Micro-computed tomography}

3-D representations of the scaffolds were obtained by using micro-computed tomography ( $\mu$-CT 20, SCANCO Medicals, Switzerland). Scanner settings were $40 \mathrm{keV}$ and $248 \mu \mathrm{A}$. Entire scaffolds were scanned in slices of $7 \mu \mathrm{m}$ thickness. CT Analyser and CT Vol Realistic 3D 
Visualization (SkyScan, Belgium) softwares were used for image processing in CT reconstructions, and in the visualization of the 3-D representations.

\subsection{Loading nanocapsules onto PCL scaffolds}

Scaffolds were treated with oxygen plasma at $50 \mathrm{~W}$ for 1 min (Advanced Plasma Systems Inc., USA) to change the hydrophobicity of the fibers and also to remove any remnants of extrusion formed in the Bioplotter.

Dry nanocapsules were suspended in $1 \%(\mathrm{w} / \mathrm{v})$ alginic acid solution at a concentration of $20 \mathrm{mg} / \mathrm{ml}$ for PLGA and $100 \mathrm{mg} / \mathrm{ml}$ for PHBV nanocapsules. After $\mathrm{O}_{2}$ plasma treatment of the scaffolds, $100 \mu \mathrm{l}$ of the nanocapsule suspension was placed onto one side of the scaffold (diameter $5 \mathrm{~mm}$, height $2.5 \mathrm{~mm}$ ) and was allowed to dry at room temperature. After drying, another $100 \mu \mathrm{l}$ of suspension was applied onto the other side of the scaffold. In total, $40 \mathrm{ng}$ of BMP loaded nanoparticles were incorporated per scaffold for all delivery conditions (single, simultaneous or sequential delivery of BMP-2 and BMP-7). The scaffolds were finally dipped into ethanol prior to $1 \mathrm{~h}$ incubation in $5 \%(\mathrm{w} / \mathrm{v}) \mathrm{CaCl}_{2}$ to crosslink the alginic acid and dried at room temperature. The constructs were ethylene oxide (EtO) sterilized (Steri-Vac gas sterilizer $5 \mathrm{XL}, \mathrm{T}=37^{\circ} \mathrm{C}$, $\mathrm{t}=4 \mathrm{~h} 45 \mathrm{~min}$ ) prior to in vitro experiments.

\subsection{In situ release studies}

In the studies of release kinetics of growth factors from the nanoparticles in the constructs, BSA was used as a model molecule. Scaffolds incorporated with BSA loaded nanocapsules were put into the 24 well plates, $1 \mathrm{ml}$ of sterile PBS was added into each well and incubated at $37^{\circ} \mathrm{C}$. At various time points $(1,3,5,10,15$ and 25 days), the medium was removed and the released protein amount was determined spectrophotometrically by using Coomassie Plus Assay (Pierce, USA). Briefly, $150 \mu \mathrm{l}$ of the sample was put into the 96 well plate and $150 \mu \mathrm{l}$ of Bradford reagent was added onto these wells. After $10 \mathrm{~min}$ at room temperature, the absorbance at $595 \mathrm{~nm}$ was determined using a plate reader (Molecular Devices, USA). The absorbance was correlated with the protein concentration by a calibration curve. Then, $1 \mathrm{ml}$ of fresh, sterile PBS was added to the wells and incubation was continued. Experiments were carried out in triplicate.

\subsection{In vitro studies}

Bone marrow MSCs were isolated from 6 week old, male Sprague-Dawley rats. The rats were euthanized and their femurs and tibia were excised, washed with DMEM containing $1000 \mathrm{U} / \mathrm{ml}$ penicillin and $1000 \mu \mathrm{g} / \mathrm{ml}$ streptomycin under aseptic conditions. The marrow in the midshaft was flushed out with DMEM containing 20\% FBS, $100 \mathrm{U} / \mathrm{ml}$ penicillin and $100 \mu \mathrm{g} / \mathrm{ml}$ streptomycin. The cells were centrifuged at $500 \times \mathrm{g}$ for $5 \mathrm{~min}$, and the resulting cell pellet was resuspended and plated in T-75 flasks. These primary cultures were incubated for 2 days. The hematopoietic and other unattached cells were removed from the flasks by repeated washes with phosphate buffered saline (PBS) (10 mM, pH 7.4) and the medium of the flasks was renewed every other day until confluency. These primary cultures were then stored in liquid nitrogen until use. EtO sterilized constructs incorporating nanoparticles were then seeded with these cells at a density of 50,000 cells/scaffold. The viable cell number during cell seeding was determined with the Nucleocounter (Chemometec, Denmark). Incubation was performed at $37^{\circ} \mathrm{C}$ and $5 \% \mathrm{CO}_{2}$ in DMEM supplemented with $10 \% \mathrm{FBS}, 10 \mathrm{mM} \beta$-glycerophosphate, $50 \mu \mathrm{g} / \mathrm{ml}$ L-ascorbic acid, $10 \mathrm{nM}$ dexamethasone and penicillin/streptomycin/amphotericin B. Viable cell number was assessed with Alamar Blue assay (USBiological). ALP activity was determined by using Randox kit (USA) where the absorbance of p-nitrophenol formed from p-nitrophenyl phosphate was measured at $405 \mathrm{~nm}$.

\subsection{Actin filament staining}

At the end of 21 days of incubation, cell seeded PCL constructs were rinsed with PBS ( $\mathrm{pH}$ 7.4) in order to remove the media, treated with paraformaldehyde (4\%) for $30 \mathrm{~min}$ at room temperature for fixation, and then with Triton X-100 (0.1\%) for 5 min to permeabilize the cell membranes. After washing with PBS, samples were incubated at $37^{\circ} \mathrm{C}$ for $30 \mathrm{~min}$ in BSA solution (1\% in PBS) solution before staining to prevent non-specific binding. After washing with a diluted BSA solution ( $0.1 \%$ in PBS), the scaffolds were stained with FITC-labeled phalloidin (1:100 dilution of the stock) for actin filaments. After several washings with PBS to remove the unbound stain, the samples were studied with the confocal laser scanning microscope (CLSM) (Leica TCS SPE, Germany) with a $488 \mathrm{~nm}$ laser for FITC-phalloidin.

\subsection{Scanning electron microscopy}

The structure of the scaffolds, constructs incorporated with nanocapsules and the cell attachment on the fiber surfaces after 21 days of incubation were studied by Scanning Electron Microscopy (SEM) after sputter coating with gold (Leica Cambridge S360, Germany). Cell seeded scaffolds were fixed after 21 days of incubation with glutaraldehyde (2.5\% in cacodylate buffer, $\mathrm{pH} 7.4$ ) for $2 \mathrm{~h}$ and then washed with cacodylate buffer several times and lyophilized prior to SEM examination. 


\section{Results}

3.1 Incorporation of the delivery system onto PCL scaffolds

We had previously reported the in situ and in vitro effect of 3-D fiber organization on the structural and mechanical properties of the PCL scaffolds and on rat bone marrow MSC proliferation and differentiation, respectively, using 3-D plotted basic (B), basic-offset (BO), crossed (C), and crossed-offset (CO) structures [19]. In the present study, two of these structures (B and $\mathrm{BO}$ ) and one randomly oriented PCL scaffold (R) produced by wet spinning were loaded with nanoparticles that can provide sequential delivery of BMP-2 and BMP-7 to further investigate the effect of growth factors and 3-D fiber organization on rat bone marrow MSC attachment, proliferation and differentiation.

The nanoparticulate delivery system based on PLGA nanocapsules carrying BMP-2 and PHBV nanocapsules carrying BMP-7 to provide a rapid release of BMP-2 and a slower release of BMP-7 was previously reported [16]. Release profile of BMP had revealed that BMP-2 was released during the initial days of the incubation while BMP-7 release was achieved in a gradual manner during the first 10 days, just like in nature. The effectiveness of this system in delivering BMP-2 and BMP-7 in sequential manner and maximizing the osteogenic activity of MSCs were previously shown in both free nanoparticulate form and upon adsorption onto chitosan scaffolds [16-18].

In the present study, nanocapsules were adsorbed onto the PCL scaffolds and entrapped under a thin coat of alginate. SEM micrographs revealed the nanocapsules under the thin layer of alginic acid coat (ca. $5 \mu \mathrm{m}$ ), that did not significantly affect the porosity of the scaffolds (Fig. 1).

PLGA and PHBV nanocapsule carrying PCL scaffolds were incubated under normal culture conditions $\left(37^{\circ} \mathrm{C}, 5 \%\right.$ $\mathrm{CO}_{2}$ ) for 21 days and were then examined with SEM for signs of detectable change in the structure. It was observed that the nanocapsules were still present on the fiber surfaces of scaffold B at the end of 21 days even though the alginic acid layer was substantially removed (data not shown).

\subsection{The release kinetics}

Release of BSA, the protein used to serve as a substitute for the growth factors, from PLGA and PHBV nanocapsules on PCL scaffolds (Fig. 2) showed that the release rate from BO was highest followed by that from free PLGA nanocapsules which was higher than nanocapsules on B and R. The highest release rate observed with $\mathrm{BO}$ impregnated nanocapsules could be due to insufficient penetration of the

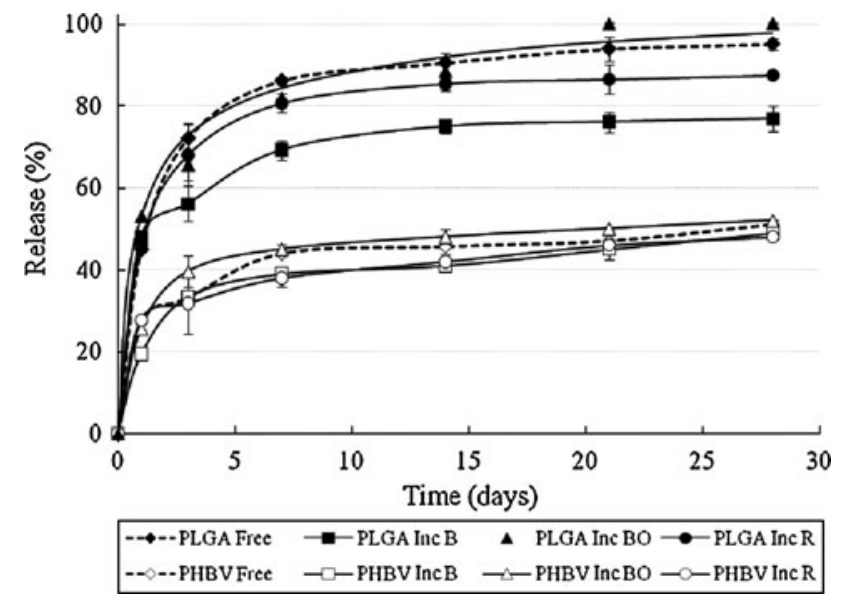

Fig. 2 BSA release from PLGA and PHBV nanocapsules incorporated in PCL scaffolds

nanocapsules to the core of the scaffold due to the tortuosity of the path in the offset form. These nanocapsules may have been located nearer to the surface without release restriction other than the thin alginic acid layer. The expected decrease in the release rates from $\mathrm{B}$ and $\mathrm{R}$ could be due to the matrix structure creating a more stagnant environment, the thin alginic acid layer covering the nanocapsules forming a barrier and also due to the long path from the core to the surface. The general trend of release in all was an immediate burst release of about 50\% of the content which then significantly slowed down leading to $70-90 \%$ release in about 3 weeks.

The release of BSA from PHBV nanocapsules followed a similar trend but the burst was much lower, around $25 \%$, and the total release in 3 weeks was about $45 \%$ for all systems with BO slightly higher than the others.

\subsection{Effect of BMP-2/BMP-7 delivery from PCL} scaffolds with different fiber orientations on the proliferation and differentiation of MSCs

After the preliminary studies using BSA as the model protein, the effect of the release mode (single, simultaneous and sequential delivery of BMP-2 and BMP-7) from nanocapsules on the PCL scaffolds was studied in vitro by using rat bone marrow MSCs.

Cell attachment and spreading on the PCL fiber surfaces were studied by fluorescence microscopy after the actin filament staining (Fig. 3) and by SEM (Fig. 4). Cells were fully spread on B, BO and R constructs. The presence of nanocapsules on the fiber surfaces can be clearly seen in the SEM micrographs.

Alamar blue test was performed to quantify cell proliferation on the scaffolds (Fig. 5a). The 3-D architecture of the PCL scaffold did not have a significant effect on the release rate of the model protein, but it affected MSC 

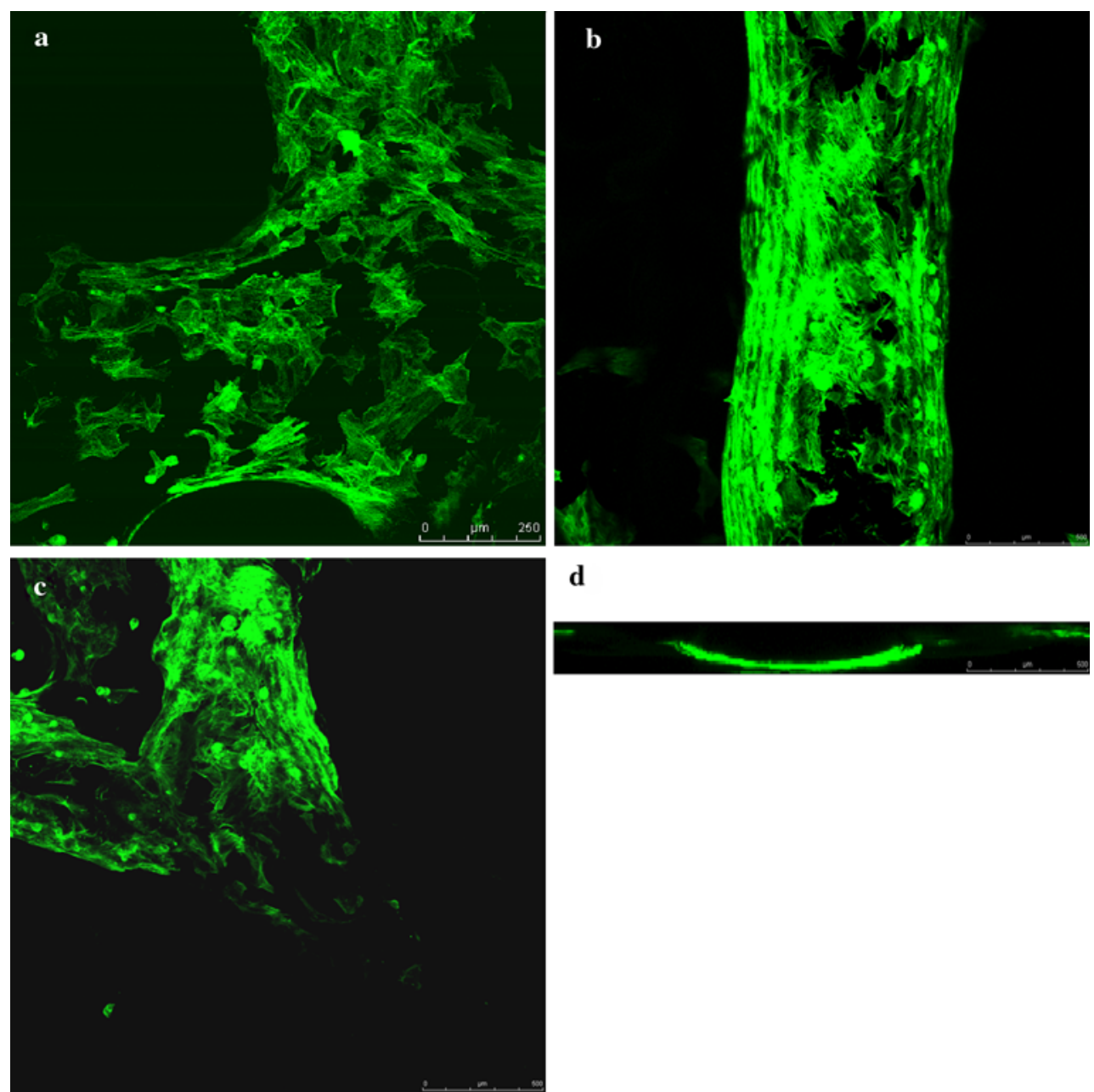

d

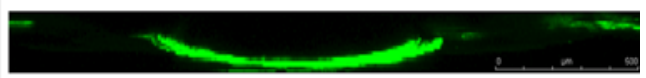

Fig. 3 Fluorescence microscopy of PCL scaffolds seeded with MSC on day 21 of incubation incorporated with PLGA and PHBV nanocapsules to provide sequential BMP-2/BMP-7 delivery.

a Scaffold B, b scaffold BO, c scaffold R, d X-section of the fiber of BO scaffold showing complete coverage of the fiber surface with MSCs. $\times 10$

proliferation. Especially after the first week, cell numbers on R scaffolds was significantly higher compared to B and $\mathrm{BO}$, probably due to the higher level of initial attachment.

When ALP activities were measured, it was observed that the ALP activity reached its highest level when BMP-2 and BMP-7 were introduced to the growth medium in a sequential manner regardless of the time or the scaffold architecture (Fig. 5b). ALP activity increased gradually in time for all scaffolds (B, BO and R) and under every delivery condition tested (single, simultaneous and sequential) during the 21 days of incubation. When the ALP activity of the various scaffold types was compared for the sequential case, it was observed that $\mathrm{R}$ scaffolds had a higher ALP activity compared to $\mathrm{B}$ and $\mathrm{BO}$, as was the case with the growth factor-free scaffolds. The ALP activity on day 21 for $\mathrm{B}, \mathrm{BO}$ and $\mathrm{R}$ were $0.767 \pm 0.016$, $0.891 \pm 0.010$ and $1.109 \pm 0.002 \mathrm{nmol} / \mathrm{min} /$ cell, respectively [19].

\section{Discussion}

Tissue engineering is a promising strategy to produce bone substitutes; however, the control of the cell organization and cell behavior to create complex, fully functional 3-D constructs has not yet been achieved. To overcome these, efforts have been concentrated on the development of multifunctional tissue engineering scaffolds capable of delivering the required bioactive agents to induce and guide cellular activities. The aim of this study was to prepare MSC seeded tissue engineered constructs consisting of PCL scaffolds with highly organized architecture impregnated with a biodegradable nanoparticulate growth factor delivery system that sequentially delivers BMP-2 and BMP-7 to mimic the natural bone healing process.

Scaffolds play a central role in the success of tissue engineering by defining the form of the construct. Since most of our tissues are highly organized and anisotropic, 

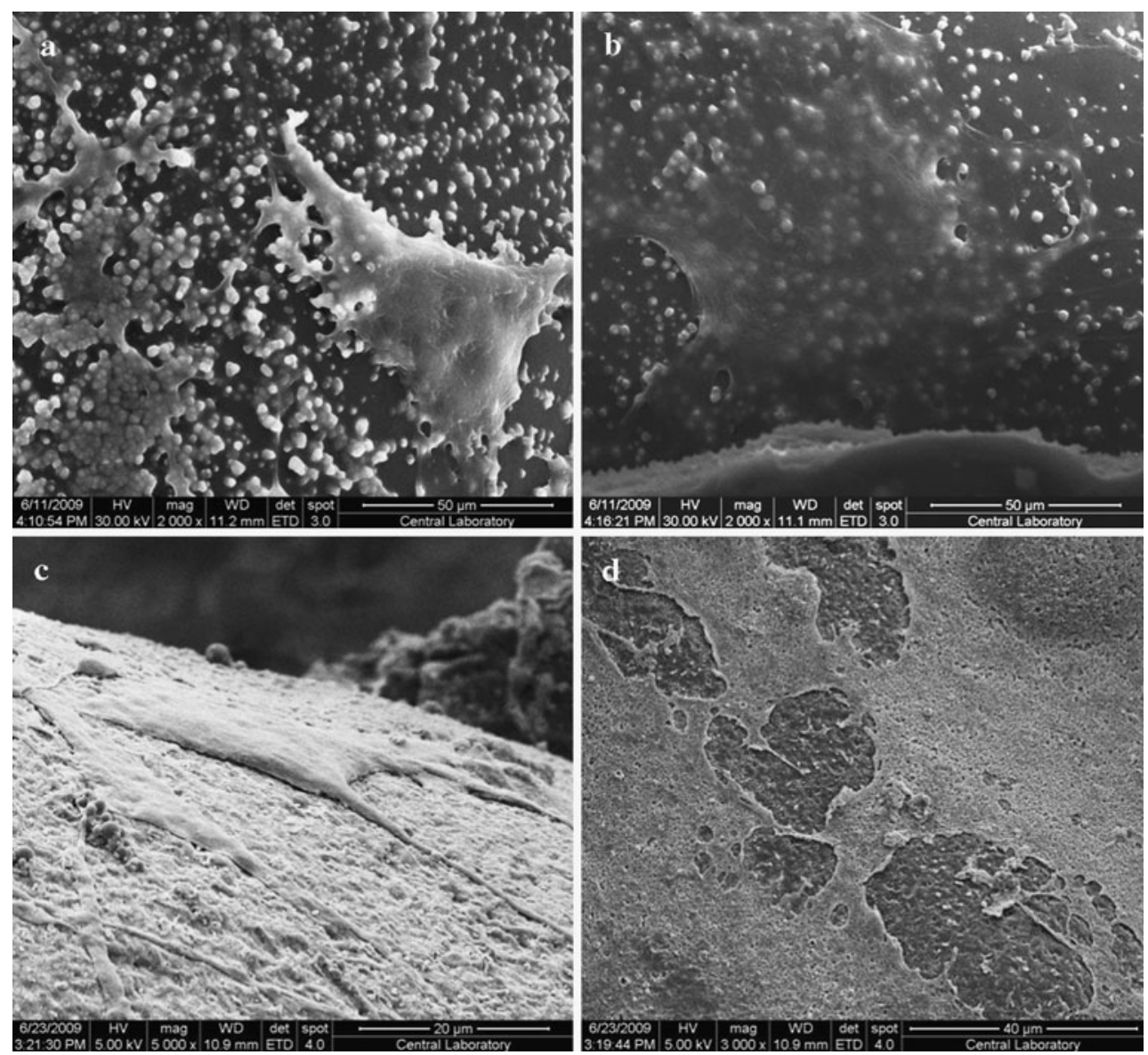

Fig. 4 SEM of MSCs on BMP-2 loaded PLGA nanocapsule incorporated PCL scaffolds a B, b BO, c, d R at the end of 21 days of culture

design of the scaffold that could mimic the natural ECM structurally and mechanically is of utmost importance. A number of techniques such as solvent casting, particulate leaching [20-22], electrospinning [23-26], fiber bonding [27], membrane lamination [28], melt molding [29], and gas foaming [30] were employed in scaffold production. However, none of these techniques can produce structures with a predefined architecture. RP technique, however, offers the capability to produce architectures that could perfectly fit into the defect site.

In the present study, PCL scaffolds produced by 3-D plotting with two different standard architectures (B, BO) and scaffolds produced by wet spinning with randomly oriented fibers (R) (Fig. 1) were used to study the effect of 3-D fiber organization on rat bone marrow MSC behavior. It was observed that random scaffolds perform better than the oriented ones (B, BO) in terms of MSC proliferation and differentiation (Fig. 5). Most of the studies in the literature investigating the effect of fiber organization involving RP techniques focused on the dependence of structural and mechanical properties of the scaffolds in relation with fiber organization and pore properties [31, 32]. In a recent study from Melchels et al. [33], the effect of pore architecture on the behavior of immortalised bone marrow derived MSCs was studied by using $\mathrm{P}(\mathrm{D}, \mathrm{L} / \mathrm{LA})$ based scaffolds produced by stereolithography and salt leaching leading to scaffolds with highly oriented and random pores, respectively. As in our study, the overall porosity of the scaffolds with different pore geometries was almost the same (ca. 68\%). They reported that scaffolds produced by stereolithography had open and more accessible pores compared to the more tortuous pores of random scaffolds. This larger pore interconnectivity caused the oriented scaffold to have higher cellular attachment and proliferation in the static culture. This is to be expected because the levels of interconnectivity is different. The pore interconnectivity in this study is not different in the scaffolds used, as assessed by u-CT analysis previously [19]. Therefore, pore interconnectivity in the current case is not a property that affects cellular behavior. Thus, it must be the 3-D fiber organization that defines the geometry of sites for the cells to adhere and the distances between them must be influential on the cell adhesion, proliferation and differentiation. Since in the random scaffold the distances vary from point to point, cells must have found more sites to attach to and stretch across. 

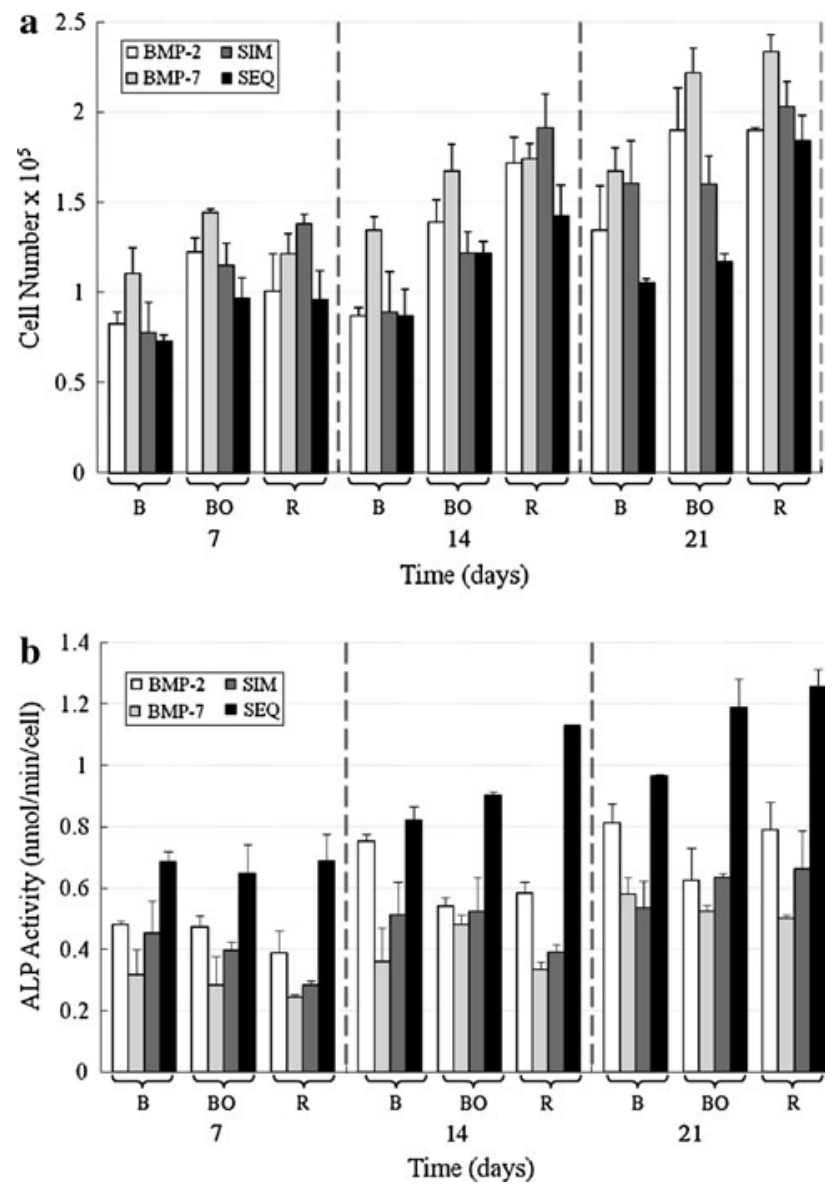

Fig. 5 a Cell proliferation on BMP loaded particle incorporated PCL scaffolds. b Specific ALP activity of MSCs on BMP loaded particle incorporated PCL scaffolds. $B$ basic, $B O$ basic-offset, $R$ random, $B M P$-2 single BMP-2 delivery from PLGA nanocapsules loaded onto scaffolds, BMP-7 single BMP-7 delivery from PHBV nanocapsules loaded onto scaffolds, SIM simultaneous BMP-2 and BMP-7 delivery (both from PLGA nanocapsules), $S E Q$ sequential BMP-2 and BMP-7 delivery (BMP-2 from PLGA and BMP-7 from PHBV nanocapsules)

The dynamic mechanical analysis of the RP PCL scaffolds used in this study were previously carried out and the scaffolds were found to exhibit a stiffness in between those of human trabecular and cortical bone [19]. In previous studies by our group, cell proliferation and the following ECM secretion were observed to enhance stiffness and ultimate tensile strength of cell seeded collagen scaffolds $[34,35]$. Thus, cell seeding and appropriate in vitro conditions during the tissue engineering process are expected to improve the mechanical properties of PCL based scaffolds and make them more compatible with the cortical bones.

The scaffolds with random and oriented fibers were made to carry a delivery system to further improve its function by mimicking the biological guidance provided by the microenvironment during healing. For this, a model protein, BSA, and two osteogenic growth factors were encapsulated in polymeric nanocapsules and loaded onto the fibers of the scaffolds under a thin alginate layer. BSA was used as a model compound because it is a protein comparable in size especially to BMP-7 (BSA: $65 \mathrm{kDa}$, BMP-7: $49 \mathrm{kDa}$, BMP-2: $18 \mathrm{kDa}$ ), and has a similar pI value (BSA: 5.5, BMPs: 5.0), indicating that it would have the same charge as the growth factors under the test conditions ( $\mathrm{pH}$ 7.4). Thus, it is an appropriate molecule to substitute the growth factors in the release studies. Also, in our previous study [16], same polyester nanocapsules (without the scaffold) loaded with BSA and BMPs presented similar release kinetics. It was observed that the release rate of BSA from PLGA nanocapsules was higher in comparison to its PHBV counterpart (Fig. 2), therefore, the nanoparticles were capable of constituting the early and late release components of a two-component sequential delivery system. Similarly, Biondi et al. [36] used BSA as a model protein encapsulated in PLGA microspheres which were then embedded in a collagen-hyaluronic acid (HA) semi-interpenetrating networks (semi-IPNs).

The studies in the literature concerning the incorporation of various growth factors into tissue engineering scaffolds generally involve mixing of the polymer with the growth factors during the preparation phase, thus, forming scaffolds that carry the factor embedded within the polymer matrix as in a monolithic device $[8,37]$. Therefore, the control on the release rate is not very precise in this case as the release of the agent is dependent on the degradation of the scaffold or solubilization of the drug molecules starting from the surface. In other studies, growth factors were loaded into large microparticles, which upon incorporation in the scaffold affected its structure and properties [9-11, 17]. In the study of Patel et al. [38], VEGF and BMP-2 were encapsulated in gelatin microparticles of ca. $30 \mu \mathrm{m}$, therefore, blocking most the pores of the scaffold that is essential for cell seeding, proliferation and also for oxygen and nutrient delivery. In another study [11], BMP-2 and IGF-1 (alone or together) were encapsulated in glycidyl methacrylated dextran/gelatin microparticles and embedded in the porous scaffold made from the same polymeric material. Sustained release from the constructs was shown, however, it was clear in the SEM micrographs that the porosity of the scaffold was considerably decreased due to the presence of the microparticles. In the present study, growth factors were encapsulated in nanocapsules which were then introduced to PCL scaffolds without affecting the structural properties of the scaffold (Fig. 1). Moreover, by maintaining the nanoparticles on the surface of the fibers rather than within the fibers, the release from the particles was not dependent on scaffold degradation or growth factor solubilization and could be fine tuned by changing the nanoparticle properties and loading conditions. 
The proliferation of MSCs and their differentiation into osteoblasts after induction with BMP-2 and BMP-7 sequentially released from the PCL scaffolds was studied. Fluorescence microscopy and SEM analysis revealed proper spreading of the cells on the fiber surfaces in the presence of nanoparticles (Figs. 3, 4). Cell proliferation was observed to have a similar trend to that of the previous results reported with free nanocapsules [16] and chitosan scaffolds [18] (Fig. 5a). Release of BMP-2 suppressed MSC proliferation when compared to the unloaded PCL scaffolds (the number of cells on day 21 for unloaded $\mathrm{B}$, $\mathrm{BO}$ and $\mathrm{R}$ scaffolds were $2.22 \pm 0.12 \times 10^{5}, 2.62 \pm$ $0.57 \times 10^{5}$ and $2.75 \pm 0.33 \times 10^{5}$, respectively [19]) and the suppression was higher than with BMP-7. In all three BMP-2 carrying systems proliferation was suppressed. The most suppressed was the BMP-2/BMP-7 sequential delivery system. This trend did not change during the 3-week test period. The ALP activity trend is also similar to our earlier results [16, 18]; the highest ALP activity, the indication of MSC differentiation, was observed in the case of sequential delivery of the growth factors (Fig. 5b). Similar results were obtained by other researchers as well, where the co-administration of BMP-2 and TGF- $\beta 3$ [8], BMP-2 and VEGF $[9,10]$ and BMP-2 and IGF-1 led to enhanced bone formation while the effect of a single growth factor was negligible.

An interesting result of this study was that the use of $R$ scaffolds seemed to perform better in housing higher cell numbers than the oriented scaffolds (B and BO) (Fig. 5). This might be due to the higher initial cell attachment on the $\mathrm{R}$ fibers due to the tortuosity of the path during cell seeding which led to the higher cell numbers on this scaffold for the rest of the in vitro culture. This result might also indicate that the scaffold design should mimic the natural ECM structure and that ordered structures are not that favored by the biological system. Besides, the distances between the organized fibers might have been too large. Therefore, scaffold architecture was influential on cell response, in terms of cell attachment and proliferation. Furthermore, cell differentiation, as measured by ALP activity, appears to be also affected by scaffold structure as well as by the delivery mode of the growth factors.

\section{Conclusion}

The positive effect of co-administration of BMP-2 and BMP-7 in a sequential manner as in nature was reflected as increased osteogenic activity. The 3-D architecture of the scaffolds affected MSC proliferation and differentiation, and scaffold with randomly organized fibers performed better than ordered PCL scaffolds. As a conclusion, it is possible to obtain multi-functional bioactive tissue engineering scaffolds with different architectures capable of delivering osteogenic factors in a number of ways and using this information it will be possible to control the level and rate of maturation of a tissue engineered construct.

Acknowledgments This project was conducted within the scope of the EU FP6 NoE Project Expertissues (NMP3-CT-2004-500283). We acknowledge the support to PY through the same project in the form of an integrated $\mathrm{PhD}$ grant. We also would like to acknowledge the support from Scientific and Technical Research Council of Turkey (TUBITAK) through project METUNANOBIOMAT (TBAG 105T508).

\section{References}

1. Lam CXF, Mo XM, Teoh SH, Hutmacher DW. Scaffold development using 3D printing with a starch-based polymer. Mater Sci Eng C. 2002;20:49-56.

2. Yeong WY, Chua CK, Leong KF, Chandrasekaran M. Rapid prototyping in tissue engineering: challenges and potential. Trends Biotechnol. 2004;22:643-52.

3. Moroni L, de Wijn JR, van Blitterswijk CA. 3D fiber-deposited scaffolds for tissue engineering: influence of pores geometry and architecture on dynamic mechanical properties. Biomaterials. 2006;27:974-85.

4. Allori AC, Sailon AM, Warren SM. Biological basis of bone formation, remodeling, and repair-part I: biochemical signaling molecules. Tissue Eng Part B. 2008;14:259-73.

5. Urist MR. Bone: formation by autoinduction. Science. 1965;150: 893-9.

6. Wang EA, Rosen V, D’Alessandro JS, Bauduy M, Cordes P, Harada T, et al. Recombinant human bone morphogenetic protein induces bone formation. Proc Natl Acad Sci USA. 1990;87: $2220-4$.

7. Cowan CM, Soo C, Ting K, Wu B. Evolving concepts in bone tissue engineering. Curr Top Dev Biol. 2005;66:239-85.

8. Simmons CA, Alsberg E, Hsiong S, Kim WJ, Mooney DJ. Dual growth factor delivery and controlled scaffold degradation enhance in vivo bone formation by transplanted bone marrow stromal cells. Bone. 2004;35:562-9.

9. Patel ZS, Young S, Tabata Y, Jansen JA, Wong MEK, Mikos AG. Dual delivery of an angiogenic an osteogenic growth factor for bone regeneration enhances in a critical size defect model. Bone. 2008;43:931-40.

10. Young S, Patel ZS, Kretlow JD, Murphy MB, Mountziaris PM, Baggett LS, et al. Dose effect of dual delivery of vascular endothelial growth factor and bone morphogenetic protein-2 on bone regeneration in a rat critical-size defect model. Tissue Eng Part A. 2009;15:2347-62.

11. Chen FM, Chen R, Wang XJ, Sun HH, Wu ZF. In vitro cellular responses containing two microencapsulated growth factors. Biomaterials. 2009;30:5215-24.

12. White AP, Vaccaro AR, Hall JA, Whang PG, Friel BC, McKee MD. Clinical applications of BMP-7/OP-1 in fractures, nonunions and spinal fusion. Int Orthop. 2007;31:735-41.

13. McKay WF, Peckham SM, Badura JM. A comprehensive clinical review of recombinant human bone morphogenetic protein-2 (INFUSE $^{\circledR}$ Bone Graft). Int Orthop. 2007;31:729-34.

14. Bessa PC, Casal M, Reis RL. Bone morphogenetic proteins in tissue engineering: the road from the laboratory to the clinic, part I (basic concepts). J Tissue Eng Regen Med. 2008;2:1-13.

15. Cho TJ, Gerstenfeld LC, Einhorn TA. Differential temporal expression of members of the transforming growth factor beta 
superfamily during murine fracture healing. J Bone Miner Res. 2002;17:513-20.

16. Yilgor P, Hasirci N, Hasirci V. Sequential BMP-2/BMP-7 delivery from polyester nanocapsules. J Biomed Mater Res. 2010; 93A:528-36.

17. Basmanav FB, Kose GT, Hasirci V. Sequential growth factor delivery from complexed microspheres for bone tissue engineering. Biomaterials. 2008;29:4195-204.

18. Yilgor P, Tuzlakoglu K, Reis RL, Hasirci N, Hasirci V. Incorporation of a sequential BMP-2/BMP-7 delivery system into chitosan-based scaffolds for bone tissue engineering. Biomaterials. 2009;30:3551-9.

19. Yilgor P, Sousa RA, Reis RL, Hasirci N, Hasirci V. 3D plotted PCL scaffolds for stem cell based bone tissue engineering. Macromol Symp. 2008;269:92-9.

20. Kose GT, Kenar H, Hasirci N, Hasirci V. Macroporous poly(3hydroxybutyrate-co-3-hydroxyvalerate) matrices for bone tissue engineering. Biomaterials. 2003;24:1949-58.

21. Cao H, Kuboyama N. A biodegradable porous composite scaffold of PGA $/ \beta$-TCP for bone tissue engineering. Bone. 2010;46: 386-95.

22. Zhang $\mathrm{P}$, Hong $\mathrm{Z}, \mathrm{Yu} \mathrm{T}$, Chen $\mathrm{X}$, Jing $\mathrm{X}$. In vivo mineralization and osteogenesis of nanocomposite scaffold of poly(lactide-coglycolide) and hydroxyapatite surface-grafted with poly(l-lactide). Biomaterials. 2009;30:58-70.

23. Santos MI, Tuzlakoglu K, Fuchs S, Gomes ME, Peters K, Unger $\mathrm{RE}$, et al. Endothelial cell colonization and angiogenic potential of combined nano- and micro-fibrous scaffolds for bone tissue engineering. Biomaterials. 2008;29:4306-13.

24. Yucel D, Kose GT, Hasirci V. Polyester based nerve guidance conduit design. Biomaterials. 2009;31:1596-603.

25. Cao H, Chen X, Huang L, Shao Z. Electrospinning of reconstituted silk fiber from aqueous silk fibroin solution. Mater Sci Eng C. 2009;29:2270-4.

26. Yu HS, Jang JH, Kim TI, Lee HH, Kim HW. Apatite-mineralized polycaprolactone nanofibrous web as a bone tissue regeneration substrate. J Biomed Mater Res. 2009;88:747-54.

27. Gomes ME, Holtorf HL, Reis RL, Mikos AG. Influence of the porosity of starch-based fiber mesh scaffolds on the proliferation and osteogenic differentiation of bone marrow stromal cells cultured in a flow perfusion bioreactor. Tissue Eng. 2006;12: 801-9.
28. Mikos AG, Sarakinos G, Leite SM, Vacanti JP, Langer R. Laminated three-dimensional biodegradable foams for use in tissue engineering. Biomaterials. 1993;14:323-30.

29. Se HO, Soung GK, Jin HL. Degradation behavior of hydrophilized PLGA scaffolds prepared by melt-molding particulateleaching method: comparison with control hydrophobic one. J Mater Sci Mater Med. 2006;17:131-7.

30. Almirall A, Larrecq G, Delgado JA, Martínez S, Planell JA, Ginebra MP. Fabrication of low temperature macroporous hydroxyapatite scaffolds by foaming and hydrolysis of an $\alpha$-TCP paste. Biomaterials. 2004;25:3671-80.

31. Lipowiecki M, Brabazon D. Design of bone scaffolds structures for rapid prototyping with increased strength and osteoconductivity. Adv Mater Res. 2010;83-86:914-22.

32. Li JP, de Wijn JR, van Blitterswijk CA, de Groot K. The effect of scaffold architecture on properties of direct 3D fiber deposition of porous Ti6Al4V for orthopedic implants. J Biomed Mater Res. 2010;92A:33-42.

33. Melchels FPW, Barradas AMC, van Blitterswijk CA, de Boer J, Feijen J, Grijpma DW. Effects of the architecture of tissue engineering scaffolds on cell seeding and culturing. Acta Biomater. 2010. doi:10.1016/j.actbio.2010.06.012.

34. Vrana NE, Elsheikh A, Builles N, Damour O, Hasirci V. Effect of human corneal keratocytes and retinal pigment epithelial cells on the mechanical properties of micropatterned collagen films. Biomaterials. 2007;28:4303-10.

35. Zorlutuna P, Elsheikh A, Hasirci V. Nanopatterning of collagen scaffolds improve the mechanical properties of tissue engineered vascular grafts. Biomacromolecules. 2009;10:814-21.

36. Biondi M, Indolfi L, Ungaro F, Quaglia F, La Rotonda MI, Netti PA. Bioactivated collagen-based scaffolds embedding proteinreleasing biodegradable microspheres: tuning of protein release kinetics. J Mater Sci Mater Med. 2009;20:2117-28.

37. Raiche AT, Puleo DA. In vitro effects of combined and sequential delivery of two bone growth factors. Biomaterials. 2004;25:677-85.

38. Patel ZS, Yamamoto M, Ueda H, Tabata Y, Mikos AG. Biodegradable gelatin microparticles as delivery systems for the controlled release of bone morphogenetic protein-2. Acta Biomater. 2008;4:1126-38. 